Revue scientifique sur la conception et l'aménagement de l'espace

\title{
Paysages des franges urbaines en projet
}

Présentation du dossier

\section{Monique Toublanc, Sophie Bonin et Pierre Dérioz}

\section{(2) OpenEdition}

1 Journals

Édition électronique

URL : http://journals.openedition.org/paysage/10362

DOI : $10.4000 /$ paysage. 10362

ISSN : 1969-6124

\section{Éditeur :}

École nationale supérieure du paysage de Versailles-Marseille, Institut national des sciences appliquées Centre Val de Loire - École de la nature et du paysage, École nationale supérieure d'architecture et de paysage de Bordeaux, École nationale supérieure d'architecture et de paysage de Lille, Agrocampus Angers

\section{Référence électronique}

Monique Toublanc, Sophie Bonin et Pierre Dérioz, «Paysages des franges urbaines en projet », Projets de paysage [En ligne], 13 | 2015, mis en ligne le 31 décembre 2015, consulté le 23 septembre 2020. URL : http://journals.openedition.org/paysage/10362 ; DOI : https://doi.org/10.4000/paysage.10362

Ce document a été généré automatiquement le 23 septembre 2020.

Projets de paysage 


\title{
Paysages des franges urbaines en projet
}

\author{
Présentation du dossier
}

Monique Toublanc, Sophie Bonin et Pierre Dérioz

1 «Les paysages des franges urbaines en projet» constituent un champ de recherche indissociable des dynamiques d'urbanisation et de métropolisation qui caractérisent la France depuis plusieurs décennies. Les espaces périurbains, longtemps en marge du champ de vision des acteurs fabriquant la ville, sont aujourd'hui regardés de façon plus complexe et polysémique qu'auparavant, à travers l'expression de franges urbaines. La revue Projets de paysage cherche ici à prendre la mesure de ce changement en donnant la parole à des chercheurs mais aussi à des professionnels de l'aménagement œuvrant sur le terrain. Tous ont communiqué au colloque «Paysages des franges urbaines. Décrire, habiter, gouverner » qui s'est tenu à Narbonne au palais des Archevêques du 5 au 7 novembre 2014', en particulier dans l'axe "gouverner» (d'autres supports de valorisation sont prévus pour les autres axes).

2 Outre une mise en projet et en paysage, ce processus de reconnaissance passe aussi par une mise en mots, dont le vocabulaire de la planification urbaine donne des signes manifestes. Dès la fin du xix ${ }^{e}$ siècle, différents concepts aujourd'hui bien connus comme ceux de «ceinture verte» (Roussel), «coulée verte», " espaces libres» abordent la question de la bordure externe de la ville.

Depuis lors, les termes appliqués à ces bords et les objets spatiaux que le vocable «frange urbaine " désigne se sont diversifiés, enregistrant les transformations de la ville et de la société. Selon l'échelle considérée, celui-ci renvoie à plusieurs situations de juxtaposition. Sous l'effet d'une extension continue et diffuse de l'urbanisation, très au-delà du pôle urbain (Bories), l'expression «franges urbaines» qualifie des configurations spatiales, correspondant à des territoires d'interface aux caractéristiques rurales ou naturelles, situés entre des espaces urbanisés à vocation résidentielle, des espaces accueillant des activités économiques industrielles (Menguy), ou commerciales, comme les plateformes logistiques pour les marchandises (Raffin, Luginbühl et Pollet). On peut ajouter à cette liste les accotements des nombreuses 
infrastructures linéaires (autoroutes, rails, canaux...) qui zèbrent en tous sens les aires urbaines (Delbaere et Ehrmann). Enfin, l'expression est également déclinée à l'échelle de la ville dense. Il s'agit alors d'interstices qui séparent les entités sociospatiales composant la ville: dénommées «intra-urbaines», les franges prennent souvent la forme de délaissés sans fonctions ou usages établis, mais n'en sont pas moins revendiquées comme des éléments constitutifs de la ville dense (Bailly). On l'aura compris les franges urbaines présentent un haut degré de variabilité et de diversité spatiales dont le seul point commun est d'être des entre-deux en passe de s'autonomiser. À cet égard, les travaux de chercheurs mais aussi d'experts praticiens (au sens de paysagistes, architectes, urbanistes...) font exister les franges urbaines comme étendues autonomes, si ce n'est dans les faits, au moins dans les discours.

4 Corrélativement à cette évolution d'une frange linéaire pensée comme démarcation à une frange "épaissie» pensée comme connexion, une figure nouvelle, la «lisière urbaine ", est apparue, construite par des professionnels de la conception de l'espace, notamment les paysagistes. Ce principe vise à projeter, à différentes échelles - région, métropole, commune, quartier - des espaces de transition non bâtis, entre des univers construits. Dans la dynamique de qualification à l'œuvre, ces entre-deux sont partie intégrante de la métropole, étendues identifiées pour elles-mêmes. Le succès de cette figure spatiale au contenu vertueux est incontestable dans le champ de la planification urbaine, comme canevas pour structurer le développement urbain à l'échelle du "grand territoire». Qui ne pourrait souhaiter substituer aux limites qui aujourd'hui morcellent le territoire, des lisières qui rétabliraient une continuité en devenant des lieux d'échanges et de complémentarité entre des espaces hétérogènes qui sont jusqu'à présent supposés s'ignorer? «Faire PLUS avec ce qui fonde la ceinture verte... Plus de nature, plus de porosité, plus d'usages ${ }^{2}$.» En revanche, lorsque l'on passe à l'échelle de la réalisation de projets concrets, le principe montre toutes ses limites. Promouvoir, à travers une solution spatiale, des rapports sociaux harmonieux fondés sur la rencontre entre deux territoires, certes contigus, mais souvent très différents (socialement, culturellement, économiquement), ne suffit pas pour les faire advenir.

5 Ainsi, par exemple, il n'est pas rare que les échanges entre les habitants de ces marges et les agriculteurs qui les exploitent soient inexistants ou extrêmement réduits, sinon même conflictuels. Les premiers ne voient pas nécessairement dans ces territoires agricoles des opportunités de consommation, l'approvisionnement via des circuits courts et la recherche de produits locaux étant bien plus souvent le fait d'habitants des centres-ville (Nikolli, Le Gall, Laval). Ce cas évoqué est à relativiser sans doute, mais il donne à penser que les obstacles sont nombreux à une traduction opérationnelle des projets de lisière. Ce sont alors les populations installées dans ces franges qui individuellement agissent et fabriquent des limites matérielles qui leur conviennent. Elles dessinent à leur échelle des interfaces qui jouent selon les cas sur la perméabilité visuelle, sur l'accessibilité physique à travers l'aménagement d'ouvertures, sur l'intégration au jardin privé de l'espace environnant, qui devient partie prenante de l'espace privatif, au moins dans l'imaginaire (Bouisset et Degrémont).

6 Ces processus d'appropriation spontanés engendrent une limite dont la physionomie d'ensemble est assez imprévisible, oscillant entre hétérogénéité et uniformité. Dans tous les cas, il est rare qu'elle soit conforme à la figure paysagiste de la lisière. Pour autant, la question reste entière: quelles solutions pourrions-nous imaginer pour établir de manière effective des liens entre des univers parfois très éloignés? Deux cas 
de figure de mise en œuvre d'une lisière sont relatés dans ce dossier (Douence et Laplace-Treyture; Méliani) et permettent d'esquisser les facteurs de réussite. Outre la maîtrise foncière, la participation des riverains et des usagers potentiels dans des processus de fabrication collectif y apparaît comme une condition nécessaire.

\section{NOTES}

1. Ce colloque a obtenu le soutien du Medde; il a été organisé par une équipe de chercheurs (Ladyss, Larep, Espace-Dev, Gecko, Geode) dans le prolongement d'une recherche sur la thématique des franges urbaines: «Paysage des franges périurbaines - Représentations, Indicateurs, Outils ", 2012-2015 (coordonné par Richard Raymond et al., 2012-2015), - retenue dans le cadre de l'appel à recherche « Paysage et développement durable - 2 » du ministère de l'Écologie, du Développement durable et de l'Énergie (Medde).

2. Atelier parisien d'urbanisme, «La ceinture verte de Paris aux $\mathrm{xxI}^{\mathrm{e}}$ siècle. Hier, aujourd'hui, demain?», 2013.

\section{AUTEURS}

\section{MONIQUE TOUBLANC}

Monique Toublanc est sociologue, ingénieur paysagiste, maître de conférences à l'École nationale supérieure de paysage de Versailles-Marseille, Laboratoire de recherche en projet de paysage (Larep)

m.toublanc[at]ecole-paysage[dot]fr

\section{SOPHIE BONIN}

Sophie Bonin est agronome et géographe, maître de conférences à l'École nationale supérieure de paysage de Versailles, Larep.

s.bonin[at]ecole-paysage[dot]fr

\section{PIERRE DÉRIOZ}

Pierre Dérioz est géographe et maître de conférences HDR à Avignon Université, UMR Espace-Dev 228 IRD.

pierre.derioz[at]univ-avignon[dot]fr 